examine by the courtesy of the Westinghouse Brake and Saxby Signal Co., in which there is no copper backing to the gilt cuprous oxide layer, no signs of the reversal near $0 \cdot 6 \mu$ are obtained. On the other hand, these cells show a small, but definite reversal in the ultra-violet.

There is no doubt that the wave-length of reversal and the magnitude of the reverse current depend on the thickness of the gold film and also, probably, on the thickness of the oxide layer. The variability of the effect over the surface of the cell described by Messrs. Poole and Atkins suggests that the gold was much thinner than in the cells I have used. The magnitude of the reversed maximum is also apparently greater, which points in the same direction. On this point, however, I would be glad to know if the figures given have been reduced to an 'equal energy spectrum', or if they relate to the intensities obtained in a spectroscope uncorrected for dispersion or for the energy distribution in the spectrum of the source used. I am hoping to obtain, in the not too distant future, the necessary data on the spectral transparency of the gold films and of the cuprous oxide layers to provide a reasonably convincing explanation of the behaviour of these cells.

I note that Messrs. Poole and Atkins found no reversal in a silver-sputtered cell. It may be of interest to mention that with a silvered cuprous oxide cell without copper backing, also prepared by the Westinghouse Co., I have found a reversal to occur at the blue end of the visible spectrum. The cell has only recently come into my hands and I have not carried out sufficient tests to see whether any return to the original polarity occurs at still shorter wave-lengths, but the indications are that this is unlikely. These cases differ from that of the gold-oxide-copper cell inasmuch as there is here only one rectifying surface, that between the metal film and the cuprous oxide. I would have preferred to say nothing about the matter until further work had been done towards its elucidation, but as the peculiarities of rectifying cells are apparently exciting a widespread interest, it may be as well to put these cases of reversal on record also.

The National Physical Laboratory, J. GUILD.

Teddington, Middlesex.

Jan. 31.

\section{Reaction Cells in Chain Reactions}

THE simplest material chain reaction would be of the type $\mathrm{H}+\mathrm{H}_{2}$ (para) $=\mathrm{H}_{2}$ (ortho $)+\mathrm{H}$, though no evidence has yet been brought forward for its exist. ence. An energy chain would be one in which an excited molecule took the place of the hydrogen atom.

We may define the chain-length by the number of fruitful collisions made by a chain carrier between its production and disappearance, regardless of the total number of collisions. If we consider a mixture of $h$ molecules of the reactant $\mathbf{H}_{2}$, and $a$ molecules of chain stoppers (for example, an aerosol of antiknock), the average chain-length $L$ is given by the simple probability expression

$$
L=\sum_{r=1}^{r=\infty} r\left(\frac{h}{a+h}\right)^{r}\left(\frac{a}{a+h}\right) / \Sigma\left(\frac{h}{a+h}\right)^{r}\left(\frac{a}{a+h}\right),
$$

provided that the pressure is so low that the probability of collision of any two molecules is practically independent of their instantaneous position in the vessel considered.
As the pressure is raised, the movements of all the molecules are hindered by the increasing resistance to diffusion ; the probability of collision with distant molecules is smaller, and with nearby molecules greater. In particular, for reactions where the average chain-length is large, collisions with the reaction products left in the path of a chain carrier will be relatively more numerous at higher pressures. This may greatly affect the average chain-length.

If $D$ is the coefficient of diffusion of the gas, the mean square displacement of the carrier is given by $\overline{x^{2}}+\overline{y^{2}}+\overline{z^{2}}=6 D t$, where $t$ is the time elapsed since the chain began. It is thus impossible for the carrier to react with molecules originally outside a cell of radius $2(6 D t)^{\frac{1}{2}}$. If $1 / Y$ is the collision frequency, $\lambda$ the mean free path and $d$ the density, $\eta$ the viscosity, $D=\frac{1}{3} \lambda \bar{c}$ and $Y=\frac{\lambda}{\bar{c}}$ where $\bar{c}$ is the R.M.S. velocity. After $r$ collisions of any kind whatsoever, $t=r Y$ and the radius of the reaction cell is $\lambda(8 r)^{\frac{1}{2}}$ and its volume $(8)^{3 / 2} \frac{4 \pi}{3} \lambda^{3} r^{3 / 2}$. Since $\lambda d=\frac{3 \eta}{\bar{c}}$ the number of molecules in the cell is proportional to $d \times \frac{r^{3 / 2}}{d^{3}}=\frac{r^{3 / 2}}{d^{2}}$, which decreases rapidly with increasing density.

The chain carrier has a greater probability of repeated collisions with molecules near the centre of its cell, but the analysis is too long to give here. It follows, however, that if the reaction products, or even impurities, deactivate the chain carrier, or if this has a probability of spontaneous radiation, that the average chain-length will decrease as the pressure rises. The number of chains initiated per second will not in general increase sufficiently with rising pressure to compensate for the decrease in length, and we may expect the rate of such reactions to decrease with rising pressure.

It is tentatively suggested that the upper pressure limit to explosions, discovered in a number of re. actions by Hinshelwood and others, may find an explanation in the facts discussed here. It must be noted, however, that in this case we should expect vigorous stirring to increase the average chain-length, and possibly to shift the upper limit to higher pressures. No evidence is yet available on this point.

\section{Clarendon Laboratory, Oxford. Jan. 28.}

\section{A. R. UBвELOHDE.}

\section{Dissociation of Acetic Acid in Water}

In the measurements of MacInnes and Shedlovsky ${ }^{1}$ and Jeffery and Vogel ${ }^{2}$ on the conductivity of acetic acid and its salts in water, which have recently been the subject of correspondence in these columns ${ }^{3}$, the most noticeable divergence is in the values for dilute solutions of sodium acetate. Jeffery and Vogel find that their values, after deducting the conductivity of the solvent, are lower than those of MacInnes and Shedlovsky, and that a correction for hydrolysis according to the equation $\mathrm{Ac}^{\prime}+\mathrm{H}_{2} \mathrm{O} \rightleftharpoons \mathrm{HAc}+\mathrm{OH}^{\prime}$ reduces their figures still further, which leads them to the view that MacInnes and Shedlovsky's values are in error. The reason for this discrepancy is that in Jeffery and Vogel's experiments the hydrogen with which the acetate ion unites comes not from the water but from the carbonic acid present in it. The proper 